\title{
The brain in acute on chronic liver failure
}

\author{
Gavin Wright • Yalda Sharifi • Maria Jover-Cobos • \\ Rajiv Jalan
}

Received: 24 February 2014 / Accepted: 22 April 2014 /Published online: 20 May 2014

(C) The Author(s) 2014. This article is published with open access at Springerlink.com

\begin{abstract}
Acute-on-chronic liver failure (ACLF) is a newly defined clinical entity with significant morbidity and mortality ( $\sim 40-90 \%$ at 1 year dependent on need for organ support at presentation). It defines a presentation with acute severe liver injury, often with multiorgan dysfunction, on a background of previously known or unknown cirrhosis. In its severest form, it is almost indistinguishable from acute liver failure, as similarly in around $5 \%$ may rapidly progress to intracranial hypertension and cerebral oedema culminating in coma and/or death. Our understanding of such cerebral sequelae is currently limited to clinical observation, though our knowledge base is rapidly expanding since recent consensus clinical definition and guidance. Moreover, there are now animal models of ACLF and imaging modalities to better characterize events in the brain that occur with ACLF. However, as yet there has been little in the way of interventional study of this condition which are much needed. In this review we dissect existing clinical and experimental data to better characterise the manifestations of ACLF on the brain and allow for the development of targeted therapy as currently the
\end{abstract}

\author{
G. Wright $\cdot$ Y. Sharifi $\cdot$ M. Jover-Cobos $\cdot$ R. Jalan $(\varangle)$ \\ Institute for Liver and Digestive Health, Liver Failure Group, UCL \\ Institute of Hepatology, The Royal Free Hospital, Upper Third UCL \\ Medical School, Pond Street, London NW3 2PF, UK \\ e-mail: r.jalan@ucl.ac.uk \\ G. Wright \\ e-mail: gavin.wright@btuh.nhs.uk \\ Y. Sharifi \\ e-mail: yalda.sharifi@ucl.ac.uk \\ M. Jover-Cobos \\ e-mail:m.cobos@ucl.ac.uk \\ G. Wright \\ Basildon \& Thurrock University Hospitals NHS Foundation Trust \\ Nethermayne, Essex SS16 5NL, Basildon, UK
}

plethora of existing interventions were designed to treat either the effects of cirrhosis or acute liver injury independently.

Keywords ACLF brain · Hepatic Encephalopathy · Hyperammonemia

\section{Introduction}

Acute-on-chronic liver failure (ACLF) is a new clinical entity with significant morbidity and mortality. In its severest form it is almost indistinguishable from acute liver failure, aside from background cirrhosis, as similarly may rapidly progress to intracranial hypertension and cerebral oedema culminating in coma and/or death. This happens in around $5 \%$, but our understanding of such cerebral sequelae is limited as little clinical or animal model data exists to-date. In this review we dissect existing clinical and experimental data to better characterise the manifestations of ACLF on the brain and allow for the development of targeted therapy as currently the plethora of existing interventions were designed to treat either the effects of cirrhosis or acute liver injury independently.

\section{Types of liver failure}

Hepatic encephalopathy (HE) is a neuropsychiatric syndrome that may develop with advancing liver insufficiency and helps define the differing clinical syndromes of liver failure. Acute liver failure (ALF) is defined by the presence of HE within 8weeks of the initial liver insult, in the absence of chronic liver disease (e.g. cirrhosis). In its most advanced stages it is associated with intracranial hypertension and possible death from brain herniation (Clemmesen et al. 1999). In cirrhosis, HE 
typically occurs insidiously with wide ranging neuropsychiatric disturbances (e.g. psychomotor dysfunction, impaired memory, decreased reaction time, diminished attention, sensory abnormalities and poor concentration). However, there is a growing number of patients with cirrhosis presenting more acutely in a manner better represented by ALF, with acute end-organ dysfunction(s) requiring support. This new clinical entity - termed 'acute-on-chronic liver failure' (ACLF), defines acute deterioration in patients with cirrhosis that triggers cerebral and clinical changes indistinct from ALF.

One of the first attempts to define 'acute-on-chronic liver failure' (ACLF) was by the London group in 2002 who suggested that ACLF encompasses the development of cerebral and clinical changes indistinct from ALF following acute liver injury on the background of chronic liver disease (Sen et al. 2002). However, as a new clinical entity, consensus over what defines ACLF remains both topical and debated. The most accepted ACLF definition to date have been set out by:

1) the Asia-Pacific Association for the Study of Liver Disease (APASL) - Acute hepatic insult manifesting as jaundice and coagulopathy, complicated within 4 weeks by ascites and/or encephalopathy in a patient with previously diagnosed or undiagnosed chronic liver disease' (Sarin et al. 2009).

2) EASL-AASLD single topic symposium - 'Acute deterioration of pre-existing chronic liver disease, usually related to a precipitating event and associated with increased mortality at 3 months due to multi-system organ failure' (Olson et al. 2011).

\section{ACLF is distinct}

On review of existing ACLF data, there is marked variance in hard clinical outcomes such as ICU mortality (35 to $89 \%$ ) and in-hospital mortality (43 to $88 \%$ ). This disparity may directly reflect the definition used and the resultant therapeutic and management practices applied. Given differing morbidity and mortality outcomes there is a fundamental need to provide a clear distinction between 'true' ACLF and less severe decompensated chronic liver disease. In ACLF patients requiring intensive care unit (ICU) support (Olson et al. 2011), reported $53 \%$ ICU mortality (and mean 14-day hospitalization), whereas others suggest a mortality as high as $89 \%$ (Levesque et al. 2013). Higher mortality is usually indicated by the extent of organ support required at admission, with Levesque et al. reporting $89 \% 1$-year mortality in those requiring mechanical ventilation. In support of earlier ACLF literature, prognostic severity scores, need for other organ support therapy, infection and total bilirubin at ICU admission were associated with high rates of ICU mortality. They also report that of the $34 \%$ discharged from ICU, 1- year survival was still only $32 \%$; with total bilirubin $<64.5$ micromol/L and length of ventilation $>9$ days were independent indicators of poor prognosis (Levesque et al. 2013).

Given increasing shortage of ICU capacity, such mortality risk stratifiers could temper our enthusiasm to provide early support for ACLF patients if certain indicators are unfavourable at presentation. However, morbidity and mortality has improved significantly for this at risk group due to early organ support and/or restorative intervention and that targeting the earlier identification of this at-risk group at hospital admission with early high-intensity care may be more beneficial in terms of morbidity, mortality and health-economic considerations. As such development of ACLF care-bundles (covering the first 48-72-hours of admission) to aid access and application of best-practice and specialist care is warranted.

\section{The brain in ACLF}

\section{Clinical description of the brain in ACLF}

As with ALF, HE is a common manifestation of ACLF (Jalan et al. 2002) and from the pathophysiological perspective similarly may progress to intracranial hypertension and cerebral oedema (Crippin et al. 1992; Jalan et al. 1997; Donovan et al. 1998). In respect to the changes within the brain in ACLF, the first acknowledgement of this hitherto unknown phenotype as distinct from decompensated cirrhosis and ALF was reported by Jalan et al. 1997; (Jalan et al. 1997) They described the previously unreported phenomenon of acute intracranial hypertension and oedema (c.f. ALF) in four patients with cirrhosis following emergency transjugular intrahepatic portosystemic shunt (TIPS) for variceal haemorrhage, associated with a marked deterioration in liver function tests. The current explanation for this post-TIPS effect relates to increased systemic microbial load secondary to redirected portal blood into the systemic circulation with therapeutic shunting. Such changes are associated with increased nitric oxide (NO) production possibly through an iNOS dependent mechanism with severe pathophysiological effects leading to circulatory failure in the critically ill cirrhotic patient (Jalan et al. 2011).

Donovan et al. later described their experience of 12 patients presenting with acutely decompensated cirrhosis with clinical and radiological evidence of raised ICP and brain oedema. They successfully treated two patients with transplantation which resulted in clinical neurological resolution. This importantly shows a reversible component to the ACLF brain, further indicating a potential therapeutic window for intervention to target cerebral sequelae of clinical ACLF and hopefully improve morbidity and mortality in this hitherto often fatal clinical presentation (Donovan et al. 1998).

Cordoba et al. expanded on our clinical understanding of the cerebral effects of ACLF by showing in similarly 
cerebrally obtunded ACLF patients demonstrating resolution of cerebral oedema and amelioration of cerebral haemodynamics on MRI, again highlighting the potential for reversibility, similar to that seen with ALF, despite the background cirrhotic brain phenotype (García Martínez et al. 2010). Moreover, in a recent large comparative clinical study of consecutive decompensated cirrhosis $(n=138)$ versus ACLF ( $n=301)$ patients, from the CANONIC Study database, Cordoba et al. elegantly demonstrate that HE associated with ACLF predominately occurs in younger often alcoholic patients, associated with more severe liver failure and systemic inflammatory response syndrome (SIRS) with extremely poor prognosis with age, bilirubin, INR, creatinine, sodium and HE grade, independent risk factors for mortality. Conversely decompensated disease occurred more frequently in older usually inactive drinkers, without severe liver failure or SIRS and often associated with diuretic use (Cordoba et al. 2014).

There has often been debate as to how frequently brain oedema and raised ICP in ACLF occurs, but new data from Joshi et.al., indicates that less than 1 in $20(\sim 5 \%)$ of such ACLF patients formally progress to such an advanced stage (Joshi et al. 2013). This is likely to be an underestimation as the patients were not monitored and given that patients with cirrhosis have some degree of cerebral atrophy, any cerebral oedema will not result in obvious clinical manifestations of increased intracranial pressure.

Other local and systemic factors have been implicated in the pathophysiology and development of this neurological syndrome. Changes in ammonia levels and inflammatory status, along with changes in cerebral haemodynamics make up our current paradigm for HE pathogenesis but their relative contribution to progression of HE in ACLF as opposed to that seen with ALF and cirrhosis has not been clearly defined (Shawcross et al. 2011). The importance of infection/SIRS in precipitating $\mathrm{HE}$ is well described in ALF and cirrhosis, but not so in ACLF. Shawcross et.al, have recently demonstrated the somewhat expected correlation between infection/SIRS and advanced HE (grades 3-4) in cirrhotic patients admitted to ITU primarily for encephalopathy (Shawcross et al. 2011). This study also discusses the relative role of ammonia in development of HE in ACLF, as plasma ammonia was not clearly associated with advancing $\mathrm{HE}$ but most patients were hyperammonaemic.

\section{Evidence of brain oedema in ACLF}

As there is no human histopathological support for the clinical findings in ACLF, there is a reliance on interpretation of animal data in models of ACLF along with the translation of clinical data from ALF and cirrhosis patients where similarities exist. Precipitants of advanced HE in ACLF are the same triggers for general decompensated cirrhosis and it remains unclear as to why in any one patient a neurological decline to intracranial hypertension and cerebral oedema ensues. One can postulate that a shift in neurophysiology may be triggered by either 1) a more significant pathological challenge (e.g. microbial inoculate dose, massive GI bleed and/or SIRS etc.), 2) background liver capacity and/or 3) local brain neurophysiology and anatomy. This latter point has been suggested in data from rat models of ACLF around a concept of 'priming', which will be discussed later.

Pathogenic synergism ammonia and inflammation Ammonia has been shown to correlate with the severity of HE in cirrhotic patients (Ong et al. 2003), and predict brain herniation in patients with ALF (Clemmesen et al. 1999). Pathophysiologically, astrocyte metabolism of ammonia produces accumulation of osmotically active glutamine to produce brain oedema and intracranial hypertension (Haussinger et al. 1994; Cordoba et al. 1996; Tofteng et al. 2006). In patients with ALF, SIRS (often secondary to infection) correlates with advancing stages of $\mathrm{HE}$ and intracranial hypertension (Rolando et al. 2000; Vaquero et al. 2003). We have previously reported a correlation between intracranial hypertension and both circulating and brain levels of proinflammatory cytokines in ALF patients (Jalan et al. 2004; Wright et al. 2007a). In patients with cirrhosis and SIRS/infection, induced hyperammonaemia worsened neuropsychological function; this suggests pathogenic synergy between hyperammonaemia and inflammation (Shawcross et al. 2004). However, from these clinical studies it is not clear whether it is the background state of the brain in cirrhosis or the associated hyperammonaemia that predisposes to the effects of the superimposed inflammation. In a histological study of a rodent model of ACLF, Wright et al., observed that acute severe microbial (Lipopolysaccharide; LPS) challenge to bile-duct ligated (BDL) rat model of cirrhosis induced marked increase in brain water (with astrocyte and associated perivascular oedema), mimicking ACLF. However, more severe LPS-induced brain oedema was evident in naïve noncirrhotic rats with induced hyperammonaemia. Interestingly, only LPS challenged BDL rats reached pre-coma stages at 3hours despite more severe brain oedema in LPS-challenged hyperammonaemic naïve rats. This decoupling of increased brain water and preserved mental state in all rats except LPStreated cirrhotic rats suggests that factors in addition to inflammation, hyperammonaemia and resultant brain swelling contribute to the effects on consciousness found in HE.

Blood brain barrier integrity (Cytotoxic versus Vasogenic Oedema) Histological analysis indicates that increases in brain water are indistinguishable from the well-documented brain oedema seen in hyperammonaemic ALF models. However, distinct from these ALF models where the blood-brain barrier (BBB) was observed to be compromised (Traber et al. 1986; Kato et al. 1989) the anatomical integrity of the BBB is 
maintained with retention of the ionic tracer lanthanum nitrate (Molecular weight $433 \mathrm{kDa}$ ) on electron microscopy. This difference may suggest a specific phenomenon of cytotoxic oedema in cirrhotic rats compared with a combined cytotoxic and vasogenic oedema in ALF. Wright et al., also looked at possible associated pathophysiological changes in the brain physiology. The astrocytic oedema in the non-cirrhotic hyperammonaemic rats was expectedly associated with increased glutamine, reduction in myo-inositol and a marked increase in the glutamine/myo-inositol ratio in keeping with the ammonia-glutamine-brain water hypothesis. However, the mild astrocytic oedema seen in the saline-treated BDL rats was markedly less despite similar degrees of plasma and brain ammonia levels as seen with the LPS-administered BDL group. The mechanisms underpinning astrocytic swelling in cirrhotic rats are therefore unclear and possibly influenced by the effects of inflammation. Yet worsening oedema following LPS-challenge in this study did not directly correlate with plasma and brain ammonia levels or brain ammonia metabolism. These experimental data may provide some insight into why ammonia levels show no direct correlation with advanced HE (grade 4) and outcomes in the clinical ACLF study by Joshi et.al., reported earlier (Joshi et al. 2013).

Systemic versus brain inflammatory processes Uniquely this study also tied the marked SIRS (peripheral trigger) to brain inflammatory responses with increased brain tissue TNF- $\alpha$ confirming our previous observation that a systemic inflammatory response may initiate brain inflammation during liver failure despite retained anatomical barrier integrity. In BDL rats high nitric oxide levels and hyperammonaemia were further associated with ammonia-induced nitrosation of astrocytic proteins (e.g. protein tyrosine nitration -PTN). Although inflammation may act synergistically with ammonia, LPS can itself lead to nitrosation of proteins in the brain (Lee et al. 2005). This may indicate that in cirrhosis, the existent background hyperammonaemia and low-grade inflammatory cerebral milieu (Jover et al. 2006) may 'prime' the animal to the effect of subsequent endotoxaemic/inflammatory insult. This concept of pathogenic synergy fits with the clinical data (Shawcross et al. 2004).

Role of oxidative stress Whether due to local cellular (e.g. astroglial) dysfunction, or peripherally (e.g. other endorgan dysfunction and/or circulating cells like neutrophils), resultant free-radical formation and/or oxidative stress, the cellular dysfunction caused by imbalanced creation, breakdown or inhibition of reactive oxygen species (ROS) and/or antioxidants, appear equally pivotal to ammonia-induced neurotoxicity. Data from both animal and human studies also suggest potential pathogenic synergy between systemic oxidative stress, and ammonia in HE, with rising ammonia levels associated with progressive oxidative stress and also free-radical production mediated by NMDA-receptor activation and ammonia-induced mitochondrial dysfunction (Marcaida et al. 1992). This could also be a source of ROS such as peroxynitrite $\left(\mathrm{OONO}^{\circ}\right)$, itself mediating $\mathrm{NO}$-induced blood brain barrier damage and astocyte dysfunction (potentially through PTN), which has been shown in models of hyperammonaemic acute or chronic liver injury and in cell culture (Bosoi et al. 2012; Jayakumar et al. 2006; Norenberg et al. 2005; Schliess et al. 2004; Rao et al. 2001); we have similarly shown this in a LPS-challenged BDL model of ACLF (Wright et al. 2007b). Oxidative stress occurs more robustly with the acute and significant hyperammonaemic insult of ALF and ACLF (Wright et al. 2007a, b), as opposed to the more insidious, though no less profound hyperammonaemia of CLD. This is an interesting observation which again points to the importance of the chronicity of injury rather than absolute level of hyperammonaemia in the clinical phenotype of HE. The likely importance of oxidative stress in HE pathogenesis also provides a the rationale for using antioxidants (e.g. N-Acetylcysteine) to ameliorate brain sequelae associated with advanced liver disease, which has proven beneficial in animal models of HE (Guerrini 1994).

Role of cerebral haemodynamics Cerebral blood flow (CBF) is integral to the HE pathogenesis and likely directly linked to alterations in both ammonia and inflammation as they independently influence cerebral haemodynamics (discussed later). As already discussed, they appear to act synergistically, which has even been shown to influence $\mathrm{CBF}$ in non-cirrhotic rat models (Pedersen et al. 2007). Development of cirrhosis is known to engender a progressively reduced CBF (cerebral oligaemia); in contrast ALF is associated to large increases in $\mathrm{CBF}$ (cerebral hyperaemia) and eventually ICP, brain oedema, coma and/or death from brain herniation. Paradoxically CBF may increase significantly in ACLF to mimic ALF. In a seminal study of cirrhotic patients receiving TIPS for either GI bleeding or ascites, TIPS-induced endotoxaemia led to an increase in the rate of production of nitric oxide, which was associated with endothelial dysfunction and an increase in CBF (Jalan et al. 2011). However, insertion of TIPS in relatively stable cirrhotics in the absence of HE had no effect on $\mathrm{CBF}$, indicating the likely pathophysiologically distinct nature of ACLF (Iversen et al. 2011). These observation also support the hypothesis that multiple hits and brain swelling is central to the brain manifestations of ACLF. 
Role of imaging techniques Given the distinct lack of histological evidence from patients with ACLF, neuroimaging modalities allow for non-invasive assessment of brain alterations in liver failure to better establish the pathogenic mechanisms involved. As the sensitivity of MRI machines improve, it is likely in the not so distant future, this technique will provide a better understanding of HE mechanisms that may prove useful for early diagnosis, local therapy design and monitoring of cerebral complications of liver failure (Chavarria and Cordoba 2013). Nath et al., in a smaller imaging study (using diffusion tensor imaging (DTI) metrics) of 23 ACLF patients compared to healthy controls, demonstrated both intracellular and extracellular brain oedema (Nath et al. 2008). The same group further compared changes in brain osmolytes with ALF, ACLF and stable cirrhosis versus healthy controls ( $n=10$ /group) using in vivo ${ }^{1} \mathrm{H}$ magnetic resonance spectroscopy (Verma et al. 2008) demonstrating lower osmotically active myo-inositol and glutamate/glutamine levels within the brain of ACLF patients. If the astrocyte brain water-glutamine hypothesis is true, this may suggest that the extent of brain oedema in ACLF is less profound than evident with ALF, a concept consistent with the change from vasogenic to cytotoxic with advanced $\mathrm{HE}$ grade (e.g. 3-4) in ALF. Recently however, brain lactate, both a brain osmolyte and one of the most important brain energy sources, has been implicated in progression of $\mathrm{HE}$ and cerebral oedema in rat models, with rising levels associated with advance stages and a potential therapeutic target (Bosoi et al. 2013). A rise in cerebral lactate may occur due to 1) hyperlactataemia related to compromised end-organ circulation which then crosses the BBB, 2) increased glycolysis due to energy failure or impairment and/or (3) increased lactate production/release or decreased lactate utilization/uptake (Rose 2010). Regardless, aiming to reduce brain lactate by inhibiting lactate synthesis is not without concern as lactate cannot be replaced by glucose as an energy source. Any resultant lack of energy sources could lead to a worsening of the functionality of the brain, inducing worsening of the motor and memory deficits (Oria and Jalan 2013). It therefore remains likely that the increase in brain lactate is a consequence of pathophysiological mechanisms rather than its cause.

\section{Animal models of ACLF}

To-date there are limited animal models of ACLF. This is not unsurprising given that the clinical syndrome has only recently been established and still under a dynamic process of characterization. However, what is common to all reported models of ACLF is the need to initially induce chronic hepatic fibrosis/cirrhosis before induction of failure with developing pathophysiological changes reflecting the typical clinical sequelae. As expressed there has been little advances in recent years to suggest the establishment of newer models of ACLF
(Belanger and Butterworth 2005). The most validated ACLF models are:

D-Galactosamine/LPS-induced inflammatory liver injury model: This ACLF model was first characterised by Liu and colleagues (Liu et al. 2007) and since utilized in a number of notable studies (Kuhla et al. 2009; Wang et al. 2012). It involves the induction of immune liver fibrosis in rats by multi-point $0.5 \mathrm{ml}$ subcutaneous injections of human serum albumin (HSA), diluted to a concentration of $8 \mathrm{~g} / \mathrm{L}$ with physiological saline (emulsified by an equal amount of incomplete Freund's adjuvant). There is a 14-day interval between the first and second injection and 10-day interval between third and fourth. Then, a further $4 \mathrm{mg}$ HSA was injected into rat tail vein twice a week for 6 weeks. Following induced immune liver fibrosis the rats are injected with intraperitoneal D-galactosamine $(400 \mathrm{mg} / \mathrm{kg})$ combined with LPS $(100 \mu \mathrm{g} / \mathrm{kg})$ to induce secondary ALF on a background of chronic liver injury.

BDL/LPS-induced ACLF model This model has been used for some time (Harry et al. 1999) and more formally characterized as a model of ACLF by Wright and colleagues (Wright et al. $2007 \mathrm{a}, \mathrm{b}$ ) with special attention to the brain sequelae of this this condition. It involves the induction of secondary biliary fibrosis/ cirrhosis by operative BDL, performed on rats under anaesthesia (intravenous diazepam $(1 \mathrm{mg} / \mathrm{kg})$, followed by intramuscular Hypnorm $\left.^{\circledR}(150 \mu \mathrm{l} / \mathrm{kg})\right)$. It involves midline laporotomy and identification of the bile duct with 'triple' ligation and then isolation and severance of the bile duct. After post-operative recovery the BDL rats are kept under regulated and controlled conditions with free access to food and water and development of secondary biliary cirrhosis over the following 4-6 weeks with clear evidence of decompensation (e.g. jaundice, ascites). Prior to termination the BDL rats are administered LPS ( $1 \mathrm{mg} / \mathrm{kg}$ ) to induce superimposed acute liver injury/failure.

\section{Multicellular interactions, synergy, priming and immune adaptation}

Given the involvement of both systemic and regional endorgan responses, inflammation, ammonia (and amino-acid metabolism) and cerebral haemodynamics, it is important to consider how cellular interactions within the brain allow for these pathophysiological observations.

Ammonia is likely to act as the priming stimulus on the background of which inflammation may produce HE. This concept is supported by interventional studies, a good example of this is the lowering of circulating ammonia with the novel therapy ornithine phenylacetate $(\mathrm{OP})$, which protected against the brain swelling associated with liver failure (Davies et al. 2009), Ammonia is detoxified by the astrocytes in the brain and swell during hyperammonaemia due to the osmotic effect of glutamine (Haussinger et al. 2000). Astrocytes form 
an integral component of the blood-brain barrier and regulate cerebral blood flow through an arachidonic acid dependent pathway (Takano et al. 2006). This observation directly links a possible mechanism that may underlie the synergy between ammonia and inflammation in which astrocytes are the critical cells. Hyperammonaemia may therefore activate astrocytes 'unlocking' the blood-brain barrier (Jalan et al. 2007), making them susceptible to endotoxaemia through a Cyclooxygensase (COX)-dependent mechanism. There is also newer evidence proposing a role for microglia (Wright et al. 2013) and pericytes in the regulation of cerebral haemodynamics via their recently reported regulatory role on capillary vascular tone (Peppiatt et al. 2006) but their role in ACLF is still unclear.

\section{Approaches to prevention and treatment}

Ammonia, inflammation, modulation of CBF autoregulation and the precipitants of acute liver injury are central to the cerebral sequelae of ACLF and therefore, important therapeutic targets in the management paradigm. Despite numerous therapeutic approaches to the wide spectrum of neuropsychiatric presentations with liver failure, there is a need for more interventional therapies for those patients with ACLF, as with ALF. Therapies are therefore focused on this mechanisms underpinning this pathogenic mechanisms outlined above. Modulation of ammoniagenesis and inflammatory processes, without compromising global brain homeostasis or inducing systemic complications, therefore remains the key to defining new therapeutic strategies for HE.

In ACLF that progresses to cerebral oedema with intracranial hypertension and resultant coma, the need to treat the multi-organ effects of liver failure are inseparable from treating its cerebral effects. Early ventilation, intensive care unit admission and judicious use of available therapies have led to a significant decline in deaths as a result of cerebral sequelae. Treating precipitating factors is as crucial as directly aiding liver recovery by prompt and specific treatment of the cause of acute liver injury. Though ACLF may be triggered by such uncommon events as sedatives or tranquilizers, vascular occlusion (hepatic vein or portal vein thrombosis) and hepatocellular carcinoma transformation, it is paramount to manage the more common precipitants such as constipation, electrolyte and acid-base imbalance, infection, gastrointestinal bleeding and portosystemic shunts. However, general management of true ACLF requires a multi-organ approach, especially for those in need of formal organ support, given the high mortality rates. There have been no formal clinical ACLF studies directly assessing therapies targeting cerebral effects but what is understood is the applied benefit of ALF therapies, which principally influence cerebral haemodynamics to limit
ICP and brain oedema. Figure 1 summarises some of the strategies that need to be assessed in ACLF patients.

General

Ventilation Early airway maintenance and intubation prevents high carbon dioxide tension and hypoxia limiting cerebral hyperaemia (Ede et al. 1986). Good airways protection limits risk of aspiration, defective gas exchange and infection. Propofol is often preferred as the sedative because it reduces ICP (though may cause hypotension) (Wijdicks and Nyberg 2002), and due to non-hepatic metabolism does not accumulate.

Circulatory support and fluid management Like ALF, ACLF is a hyperdynamic state with high cardiac output, low mean arterial pressure (MAP), and low systemic vascular resistance. Moreover given background cirrhosis there is already generalized vasodilatation, profound neurohormonal activation and resultant vasoconstricted regional vascular beds (Jalan 2005). MAP should be maintained at a level to keep CPP between 50 and $65 \mathrm{mmHg}$ (Davies et al. 1994). Ensuing Multi-organ failure (MOF) often necessitates inotropes; in those refractory to inotropes a short synacthen test on ITU admission guides steroid use as adrenal insufficiency (common in cirrhosis) (Harry et al. 2002).

Electrolyte imbalance Hyponatraemia $\leq 125 \mathrm{mmol} / \mathrm{L}$ may precipitate cerebral oedema and is a contraindication for orthotopic liver transplant (OLT) (Jalan et al. 1997; Cordoba et al. 1998). In ALF, induced hypernatraemia may improve ICP and reduce inotropic requirements (Murphy et al. 2004), though potential benefit has not been formally assessed in ACLF patients.

Antibiotic/antimicrobial agent Given the incidence of infection/SIRS related neurological decline, empirical broadspectrum antibiotics/antifungal is a requirement and should be targeted once an organism identified (Rolando et al. 1990, 2000).

Glycaemic control Disturbed glycaemic and lipid control is common in progressive liver disease and worsened by the stress response in ACLF that could provoke neuroglycopenia and brain oedema. Tight glycaemic control using insulin reduces oxidative stress (which triggers insulin resistance), limits mitochondrial liver damage, and improves endothelial nitric oxide activation to optimize blood flow, limiting tissue injury, and improve outcomes (Langouche et al. 2005; Houstis et al. 2006).

\section{Specific}

In ALF there are many therapies utilized to lower intracranial hypertension and oedema. However such therapies (e.g. 
Fig. 1 Target Therapies for brain effects of ACLF

\section{THERAPIES FOR BRAIN EFFECTS OF ACLF}

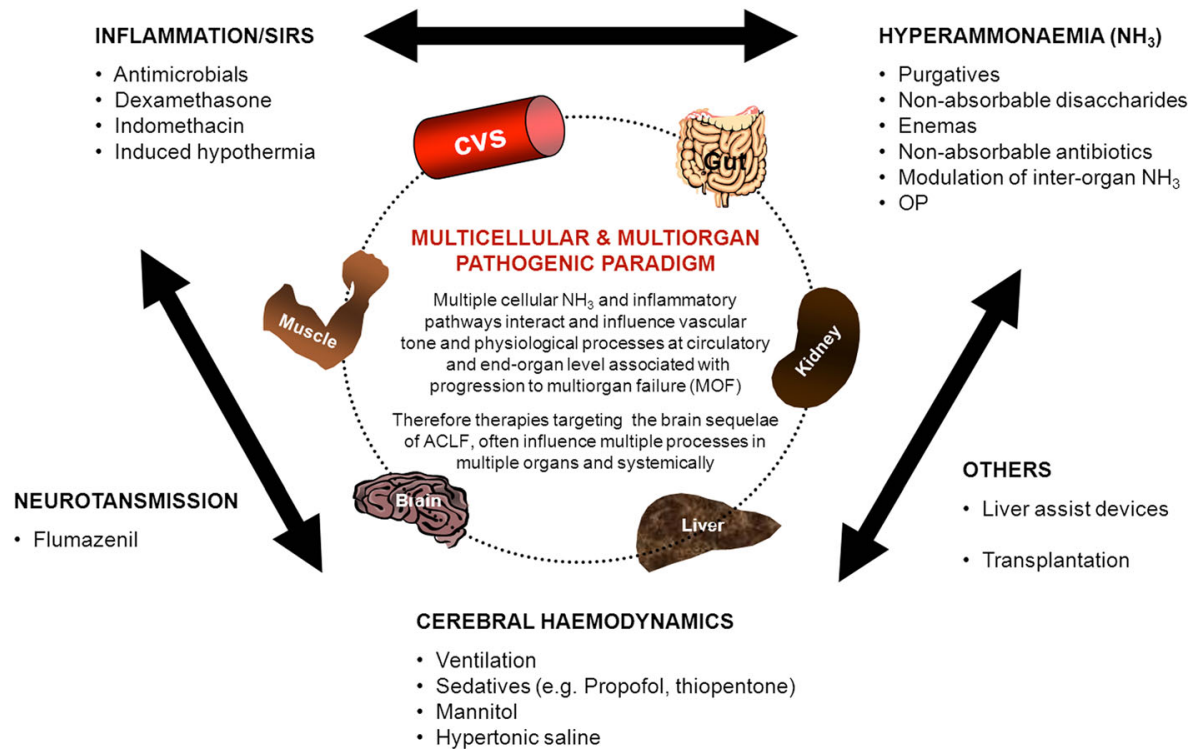

mannitol, hypothermia, hypernatraemia, anti-epileptics and indomethacin) currently have no evidence in ACLF and require evaluation in specific ACLF clinical trials. In respect to ammonia-lowering therapy, although disaccharides are useful in ALF, the data of its usefulness in ACLF patients is lacking. Otherwise none of the ammonia-lowering therapies have been trialled in this condition although as discussed previously ornithine phenylacetate may have a role but will need to be proven in appropriate clinical trials.

\section{Albumin dialysis using molecular adsorbents recirculating} system The extracorporeal device that has had the most clinical evaluation is the 'Molecular Adsorbent Recirculating System (MARS)' that provides counter-current dialysis against albumin and bicarbonate circuits. Albumin dialysis with MARS decreases retained substances and improves haemodynamics and HE. In a randomised clinical trial of MARS in ACLF patients performed in the US, there was a significant benefit of MARS in reducing the time to wake up and effectiveness. In another multinational, multicentre trial of 189 ACLF patients randomized to MARS $(n=95)$ or standard therapy (SMT) $(n=94)$, there was no significant beneficial effect of MARS on 28-day survival ( $\sim 60 \%)$. However, MARS had an acceptable safety profile, significant dialysis effect and non-significantly improved advanced HE (34) grades (Bañares et al. 2013).

\section{Conclusion}

The brain sequelae of clinical ACLF are often profound and associated with significant morbidity and mortality. As yet there has been little study in either humans or animal models of ACLF that allow us to better elucidate the histopathophysiological characteristics and define the syndrome. However, there is some understanding of managing the clinical presentation using a plethora of interventions targeting the pathogenic paradigm of ammonia, inflammation and cerebral haemodynamic dysregulation which are central to the pathogenesis of HE. A greater understanding of the interplay between interorgan ammonia and amino acid metabolism, inflammatory responses and cerebral haemodynamic in ACLF is likely to lead to the development of new therapeutic approaches.

Open Access This article is distributed under the terms of the Creative Commons Attribution License which permits any use, distribution, and reproduction in any medium, provided the original author(s) and the source are credited.

\section{References}

Bañares R, Nevens F, Larsen FS, Jalan R, Albillos A, Dollinger M, Saliba F, Sauerbruch T, Klammt S, Ockenga J, Pares A, Wendon J, Brünnler T, Kramer L, Mathurin P, de la Mata M, Gasbarrini A, Müllhaupt B, Wilmer A, Laleman W, Eefsen M, Sen S, Zipprich A, Tenorio T, Pavesi M, Schmidt HH, Mitzner S, Williams R, Arroyo V (2013) RELIEF study group. Extracorporeal albumin dialysis with the molecular adsorbent recirculating system in acute-on-chronic liver failure: the RELIEF trial. Hepatology 57(3):1153-1162

Belanger M, Butterworth RF (2005) Acute liver failure: a critical appraisal of available animal models. Metab Brain Dis 20:409-423

Bosoi CR, Yang X, Huynh J, Parent-Robitaille C, Jiang W, Tremblay M, Rose CF (2012) Systemic oxidative stress is implicated in the pathogenesis of brain edema in rats with chronic liver failure. Free Radic Biol Med 52:1228-1235 
Bosoi CR, Zwingmann C, Marin H, Parent-Robitaille C, Huynh J et al (2013) Increased brain lactate is central to the development of brain edema in rats with chronicliverdisease. J Hepatol. doi:10.1016/j.jhep.2013.10.011

Chavarria L, Cordoba J (2013) Magnetic resonance of the brain in chronic and acute liver failure. Metab Brain Dis. doi:10.1007/s11011-0139452-9

Clemmesen JO, Larsen FS, Kondrup J, Hansen BA, Ott P (1999) Cerebral herniation in patients with acute liver failure is correlated with arterial ammonia concentration. Hepatology 29:648-653

Cordoba J, Gottstein J, Blei AT (1996) Glutamine, myo-inositol, and organic brain osmolytes after portocaval anastomosis in the rat: implications for ammonia-induced brain oedema. Hepatology 24:919-923

Cordoba J, Gottstein J, Blei AT (1998) Chronichyponatremia exacerbates ammonia-induced brain edema in rats after portacaval anastomosis. J Hepatol 29:589-594

Cordoba J, Ventura-Cots M, Simón-Talero M, Amorós A, Pavesi M, Vilstrup H, Angeli P, Domenicali M, Ginés P, Bernardi M, Arroyo V (2014) CANONIC Study Investigators of the EASL-CLIF Consortium. Characteristics, risk factors, and mortality of cirrhotic patients hospitalized for hepatic encephalopathy with and without acute-on-chronic liver failure (ACLF). J Hepatol 60:275-281

Crippin JS, Gross JB Jr, Lindor KD (1992) Increased intracranial pressure and hepatic encephalopathy in chronic liver disease. Am J Gastroenterol 87: 879-882

Davies MH, Mutimer D, Lowes J, Elias E, Neuberger J (1994) Recovery despite impaired cerebral perfusion in fulminant hepatic failure. Lancet 343:1329-1330

Davies NA, Wright G, Ytrebo LM, Stadlbauer V, Fuskevag OM, Zwingmann C, Davies DC et al (2009) L-ornithine and phenylacetate synergistically produce sustained reduction in ammonia and brain water in cirrhotic rats. Hepatology 50:155-164

Donovan JP, Schafer DF, Shaw BW Jr, Sorrell MF (1998) Cerebral edema and increased intracranial pressure in chronic liver disease. Lancet 351: 719-721

Ede RJ, Gimson AE, Bihari D, Williams R (1986) Controlled hyperventilation in the prevention of cerebral oedema in fulminant hepatic failure. J Hepatol 2:43-51

García Martínez R, Rovira A, Alonso J, Aymerich FX, Huerga E, Jacas C et al (2010) A long-term study of changes in the volume of brain ventricles and white matter lesions after successful liver transplantation. Transplantation 89:589-594

Guerrini VH (1994) Effect of antioxidants on ammonia induced CNSrenal pathobiology in sheep. Free Radic Res 21:35-43

Harry D, Anand R, Holt S, Davies S, Marley R, Fernando B, Goodier D et al (1999) Increased sensitivity to endotoxemia in the bile ductligated cirrhotic rat. Hepatology 30:1198-1205

Harry R, Auzinger G, Wendon J (2002) The clinical importance of adrenal insufficiency in acute hepatic dysfunction. Hepatology 36:395-402

Haussinger D, Laubenberger J, vom Dahl S, Ernst T, Bayer S, Langer M, Gerok W et al (1994) Proton magnetic resonance spectroscopy studies on human brain myo-inositol in hypo-osmolarity and hepatic encephalopathy. Gastroenterology 107:1475-1480

Haussinger D, Kircheis G, Fischer R, Schliess F, vom Dahl S (2000) Hepatic encephalopathy in chronic liver disease: a clinical manifestation of astrocyte swelling and low-grade cerebral edema? J Hepatol 32:1035-1038

Houstis N, Rosen ED, Lander ES (2006) Reactive oxygen species have a causal role in multiple forms of insulin resistance. Nature 440:944 948

Iversen P, Keiding S, Mouridsen K, Ott P, Vilstrup H (2011) Transjugular intrahepatic portosystemic shunt does not alter cerebral blood flow. Clin Gastroenterol Hepatol 9:1001-1003

Jalan R (2005) Acute liver failure: current management and future prospects. J Hepatol 42(Suppl):S115-S123

Jalan R, Dabos K, Redhead DN, Lee A, Hayes PC (1997) Elevation of intracranial pressure following transjugular intrahepatic portosystemic stent-shunt for variceal haemorrhage. J Hepatol 27: 928-933

Jalan R, Pollok A, Shah SH, Madhavan K, Simpson KJ (2002) Liver derived pro-inflammatory cytokines may be important in producing intracranial hypertension in acute liver failure. J Hepatol 37:536538

Jalan R, OldeDamink SW, Hayes PC, Deutz NE, Lee A (2004) Pathogenesis of intracranial hypertension in acute liver failure: inflammation, ammonia and cerebral blood flow. J Hepatol 41: 613-620

Jalan RG, Davies NA, Hodges SJ (2007) L-Ornithine Phenylacetate $(\mathrm{OP})$ : a novel treatment for hyperammonemia and hepatic encephalopathy. Med Hypotheses

Jalan R, OldeDamink SW, TerSteege JC, Redhead DN, Lee A, Hayes PC et al (2011) Acute endotoxemia following transjugular intrahepatic stent-shunt insertion is associated with systemic and cerebral vasodilatation with increased whole body nitric oxide production in critically ill cirrhotic patients. J Hepatol 54:265-271

Jayakumar AR, Panickar KS, Murthy Ch R, Norenberg MD (2006) Oxidative stress and mitogen-activated protein kinase phosphorylation mediate ammonia-induced cell swelling and glutamate uptake inhibition in cultured astrocytes. J Neurosci 26:4774-4784

Joshi D, O'Grady J, Patel A, Shawcross D, Connor S, Deasy N, Willars C, Bernal W, Wendon J, Auzinger G (2013) Cerebral oedema is rare in acute-on-chronic liver failure patients presenting with high-grade hepatic encephalopathy. Liver Int. doi:10.1111/liv.12257 [Epub ahead of print] PMID: 23844567]

Jover R, Rodrigo R, Felipo V, Insausti R, Saez-Valero J, Garcia-Ayllon MS, Suarez I et al (2006) Brain oedema and inflammatory activation in bile duct ligated rats with diet-induced hyperammonemia: a model of hepatic encephalopathy in cirrhosis. Hepatology 43: $1257-1266$

Kato M, Sugihara J, Nakamura T, Muto Y (1989) Electron microscopic study of the blood-brain barrier (BBB) in rats with brain oedema and encephalopathy due to acute hepatic failure. Gastroenterol Jpn 24:135-142

Kuhla A, Eipel C, Abshagen K, Siebert N, Menger MD, Vollmar B (2009) Role of the perforin/granzyme cell death pathway in DGal/LPS-induced inflammatory liver injury. Am J Physiol Gastrointest Liver Physiol 296:G1069-1076

Langouche L, Vanhorebeek I, Vlasselaers D, Vander Perre S, Wouters PJ, Skogstrand K, Hansen TK et al (2005) Intensive insulin therapy protects the endothelium of critically ill patients. J Clin Invest 115: 2277-2286

Lee JC, Cho GS, Kim HJ, Lim JH, Oh YK, Nam W, Chung JH et al (2005) Accelerated cerebral ischemic injury by activated macrophages/microglia after lipopolysaccharide microinjection into rat corpus callosum. Glia 50:168-181

Levesque E, Saliba F, Ichaï P, Samuel D (2013) Outcome at 1-year of patients with cirrhosis admitted to ICU and requiring mechanical ventilation. J Hepatol. doi:10.1016/j.jhep.2013.11.012

Liu XH, Chen Y, Wang TL, Lu J, Zhang LJ, Song CZ, Zhang J, Duan ZP (2007) Establishment of a D-galactosamine/lipopolysaccharide induced acute-on-chronic liver failure model in rats. Zhonghua Ganzangbing Zazhi 15:771-775

Marcaida G, Felipo V, Hermenegildo C, Minana MD, Grisolia S (1992) Acute ammonia toxicity is mediated by the NMDA type of glutamate receptors. FEBS Lett 296:67-68

Murphy N, Auzinger G, Bernel W, Wendon J (2004) The effect of hypertonic sodium chloride on intracranial pressure in patients with acute liver failure. Hepatology 39:464-470

Nath K, Saraswat VA, Krishna YR, Thomas MA, Rathore RK, Pandey CM, Gupta RK (2008) Quantification of cerebral edema on diffusion tensor imaging in acute-on-chronic liver failure. NMR Biomed 21:713-722

Norenberg MD, Rao KV, Jayakumar AR (2005) Mechanisms of ammonia-induced astrocyte swelling. Metab Brain Dis 20:303-318 
Olson JC, Wendon JA, Kramer DJ, Vicente A, Jalan R, Garcia-Tsao G et al (2011) Intensive care of the patient with cirrhosis. Hepatology 54:1864-1872

Ong JP, Aggarwal A, Krieger D, Easley KA, Karafa MT, Van Lente F, Arroliga AC et al (2003) Correlation between ammonia levels and the severity of hepatic encephalopathy. Am J Med 114:188-193

Oria M, Jalan R (2013) Brain lactate in hepatic encephalopathy: Friend or foe? Journal of Hepatology (In press)]

Pedersen HR, Ring-Larsen H, Olsen NV, Larsen FS (2007) Hyperammonemia acts synergistically with lipopolysaccharide in inducing changes in cerebral hemodynamics in rats anaesthetised with pentobarbital. J Hepatol 47:245-252

Peppiatt CM, Howarth C, Mobbs P, Attwell D (2006) Bidirectional control of CNS capillary diameter by pericytes. Nature 443:700-704

Rao KV, Norenberg MD (2001) Cerebral energy metabolism in hepatic encephalopathy and hyperammonemia. Metab Brain Dis 16:67-78

Rolando N, Harvey F, Brahm J, Philpott-Howard J, Alexander G, Gimson A, Casewell M et al (1990) Prospective study of bacterial infection in acute liver failure: an analysis of fifty patients. Hepatology 11:49-53

Rolando N, Wade J, Davalos M, Wendon J, Philpott-Howard J, Williams $\mathrm{R}$ (2000) The systemic inflammatory response syndrome in acute liver failure. Hepatology 32:734-739

Rose C (2010) Increase brain lactate in hepatic encephalopathy: cause or consequence? Neurochem Int 57:389-394

Sarin SK, Kumar A, Almeida JA, Chawla YK, Fan ST, Garg H et al (2009) Acute-on chronic liver failure: consensus recommendations of the Asian Pacific Association for the Study of the Liver (APASL). Hepatol Int 3:269-282

Schliess F, Foster N, Gorg B, Reinehr R, Haussinger D (2004) Hypoosmotic swelling increases protein tyrosine nitration in cultured rat astrocytes. Glia 47:21-29

Sen S, Williams R, Jalan R (2002) The pathophysiological basis of acuteon-chronic liver failure. Liver Int 22:5-13

Shawcross DL, Davies NA, Williams R, Jalan R (2004) Systemic inflammatory response exacerbates the neuropsychological effects of induced hyperammonemia in cirrhosis. J Hepatol $40: 247-254$
Shawcross DL, Sharifi Y, Canavan JB, Yeoman AD, Abeles RD, Taylor NJ (2011) Infection and systemic inflammation, not ammonia, are associated with grade $3 / 4$ hepatic encephalopathy, but not mortality in cirrhosis. J Hepatol 54:640-649

Takano T, Tian GF, Peng W, Lou N, Libionka W, Han X, Nedergaard M (2006) Astrocyte-mediated control of cerebral blood flow. Nature Neuroscience 9:260-267

Tofteng F, Hauerberg J, Hansen BA, Pedersen CB, Jorgensen L, Larsen FS (2006) Persistent arterial hyperammonemia increases the concentration of glutamine and alanine in the brain and correlates with intracranial pressure in patients with fulminant hepatic failure. J Cereb Blood Flow Metab 26:21-27

Traber PG, Ganger DR, Blei AT (1986) Brain oedema in rabbits with galactosamine-induced fulminant hepatitis. Regional differences and effects on intracranial pressure. Gastroenterology 91:1347-1356

Vaquero J, Polson J, Chung C, Helenowski I, Schiodt FV, Reisch J, Lee WM et al (2003) Infection and the progression of hepatic encephalopathy in acute liver failure. Gastroenterology 125:755-764

Verma A, Saraswat VA, Radha Krishna Y, Nath K, Thomas MA, Gupta RK (2008) In vivo $1 \mathrm{H}$ magnetic resonance spectroscopy-derived metabolite variations between acute-on-chronic liver failure and acute liver failure. Liver Int 28:1095-1103

Wang LW, Wang LK, Chen H, Cheng F, Li X, He CM, Gong ZJ (2012) Ethyl pyruvate protects against experimental acute-on-chronic liver failure in rats. World J Gastroenterol 18:5709-5718

Wijdicks EF, Nyberg SL (2002) Propofol to control intracranial pressure in fulminant hepatic failure. Transplant Proc 34:1220-1222

Wright G, Shawcross D, Jalan R (2007a) Brain cytokine flux in acute liver failure and its relationship with intracranial hypertension. Metab Brain Dis 22:375-388

Wright G, Davies NA, Shawcross DL, Hodges SJ, Harry D, Moore KP, Mani A, Davies DC, Brooks HF, Williams R, Jalan R (2007b) Endotoxaemia produces pre-coma and brain swelling in bile duct ligated rats. Hepatology 45(6):1517-1526

Wright G, Newman TA, Sharifi Y, Davies NA, Perry H, Jalan R (2013) Characterization of temporal microglia and astrocyte immune responses in bile-duct ligated rat models of cirrhosis (In press) 